\title{
Prediction of a suitable mobile phase composition in reversed-phase high-performance liquid chromatography using fragmental constants
}

\author{
R. Wehrens ${ }^{\text {a,* }}$, L. Buydens ${ }^{a}$, H. Hindriks ${ }^{b}$, F. Maris ${ }^{c}$ \\ a Laboratory for Analytical Chemistry, Catholic University Nijmegen, Toernooiveld 1, 6525 ED Nijmegen, Netherlands \\ ${ }^{\mathrm{b}}$ AKZO Pharma Group, Diosynth International BV, P.O. Box 20, 5340 BH Oss, Netherlands \\ c AKZO Pharma Group, NV Organon, P.O. Box 20, 5340 BH Oss, Netherlands
}

Received 25 November 1993; accepted 21 February 1994

\begin{abstract}
An expert system is described that proposes an optimal mobile phase composition in reversed-phase high-performance liquid chromatography. This is achieved by predicting the retention index of the compound of interest from fragmental constants. Using a calibration line, set up with reference compounds, it is possible to infer from the retention index the percentage of organic modifier in the mobile phase that will result in a capacity factor between three and ten. The expert system can be trained to find optimal retention index values for newly defined fragments based on a set of compounds with known retention index.
\end{abstract}

\section{Introduction}

The problem of choosing experimental conditions under which to perform high-performance liquid chromatography (HPLC) experiments is large, mainly because of the large number of factors playing a role in the retention mechanism. In reversed-phase HPLC, one of the factors that is of great importance is the amount of organic modifier in the mobile phase. If the amount of organic modifier in the mobile phase is increased, the polarity of the mixture decreases, and substances will elute faster through the column. This

* Corresponding author. provides a simple mechanism to control retention times. Short retention times offer the advantage of speed, but may yield insufficient separation. Longer retention times are costly; moreover, the advantage of an increase in resolution will become small with longer retention times. A compromise must be found that leads to a good separation in an acceptable time. The problem is relevant in, for instance, the pharmaceutical industry, where HPLC is often used to test the purity of new compounds. Other compounds present in the sample may be very similar to the target compound and will therefore only be detected if the separation conditions are optimal. If a good prediction can be made of the mobile phase composition to achieve such optimal conditions, less experiments are needed to determine 
whether or not isomers or other contaminants are present, and a considerable amount of time and effort can be saved.

In this article, we present an approach to estimate the mobile phase composition in reversed-phase HPLC using a $\mu$-Bondapak C18 column, based on the molecular structure of the compound of interest. Therefore, our prediction is based solely on the information contained in the chemical structure, without use of parameters that require measurements. Our approach is to divide the molecule, represented by a connection table, into fragments that should account for some part of the functionality of the compound. The functionality of the molecule then is represented by the sum of the functionalities of the fragments that are present in the molecule. This assumption has proven to be reasonably successful in a number of applications [1].

Our goal is to predict the percentage of organic modifier in the mobile phase so that the compound of interest will have a high chance to be separated from other compounds in the sample, in a reasonably short time. A good separation can usually be achieved if the capacity factor of the compound is in the range between 3 and 10 . Our goal is a capacity factor of 5 . With larger capacity factors, the resolution increase is rather small. The capacity factor, $k^{\prime}$, is given by

$k^{\prime}=\frac{t_{\mathrm{R}}-t_{0}}{t_{0}}$

with $t_{\mathrm{R}}$ and $t_{0}$ the retention time and the void time, respectively. Direct prediction of $k^{\prime}$ is difficult because of the poor reproducibility of the capacity factor under different circumstances, e.g., due to column aging. Instead of the capacity factor also the retention index (RI) of a compound can be used. The intra- and interlaboratory reproducibility of retention indices is considerably better than that of capacity factors [2]. The retention index is calculated by comparing the capacity factors of a series of standard compounds with the capacity factor of the test compound. Baker and co-workers defined a retention index scale in HPLC, using 2-ketoalkanes as reference compounds [3], which is used in the pres- ent work. Also other reference compounds can be used [4-7]. The retention index is defined as

$$
\mathrm{RI}=100 \frac{\log k^{\prime}-\log k_{N}^{\prime}}{\log k_{N+1}^{\prime}-\log k_{N}^{\prime}}+100 N
$$

where $k^{\prime}$ indicates the capacity factor of the test compound, $k_{N}^{\prime}$ that of the reference compound eluting just before the test compound, and $k_{N+1}^{\prime}$ that of the reference just after the test compound. The retention index of a reference compound is by definition given by 100 times the carbon number $N$ in the formula.

The approach to find a suitable mobile phase composition proceeds as follows. In this study, methanol $(\mathrm{MeOH})$ is always used as organic modifier. First, the retention index of the compound of interest is calculated, using fragmental values. Because it is known that the retention-index values are slightly dependent on the modifier percentage, only RI data have been used that have been obtained with methanol percentages between $55 \%$ and $65 \%$. Then, this retention index is converted to a percentage of methanol in steps of $5 \%$ that should provide a $k^{\prime}$ of about 5 , using the graph depicted in Fig. 1. This graph has been set up using the interpolated mobile phase com-

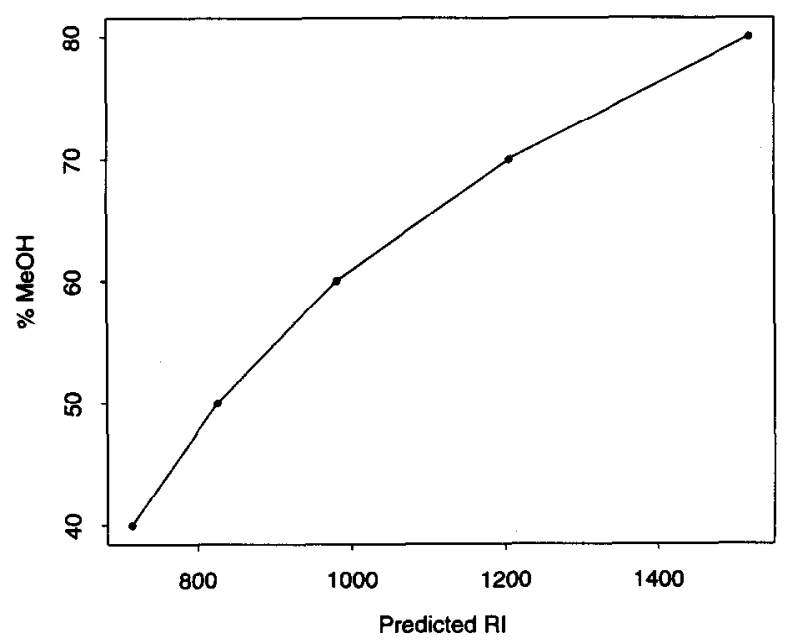

Fig. 1. Relation between retention index predicted at $60 \%$ $\mathrm{MeOH}$ and modifier percentage. Points on the line indicate the mobile phase composition where compounds with a certain retention index at $60 \% \mathrm{MeOH}$ have a capacity factor of 5. Other conditions in all experiments: $\mathrm{pH} \mathrm{7.4,} \mathrm{column:} \mu$ Bondapak C18. 
positions where the reference compounds, the 2-ketoalkanes, have a capacity factor of 5 (see Fig. 2). It is advisable to calibrate the graph of Fig. 1 periodically to prevent deviations, caused, e.g., by aging columns. These deviations are apparent when the predictions of the expert system tend to deviate from the experimental results.

The main assumption of our method is that the retention index of a molecule can be calculated as follows:

$\mathrm{RI}(\% \mathrm{MeOH})=\sum_{i} \mathrm{RI}_{f_{i}}(\% \mathrm{MeOH}) N_{f_{i}}$

with $i$ looping over all fragments present, $f_{i}$ the $i$ th fragment, and $N_{f_{i}}$ the number of times fragment $f_{i}$ is present in the molecule. $\mathbf{R I}_{f_{i}}$ is the fragmental retention index, dependent on the amount of organic modifier (in our case always $\mathrm{MeOH}$ ) in the mobile phase. If necessary, interactions between fragments may be introduced, and the formula then becomes:

$\mathrm{RI}(\% \mathrm{MeOH})=\sum_{i} \mathrm{RI}_{f_{i}}(\% \mathrm{MeOH}) N_{f_{i}}+\sum_{i, j} I_{f_{i}, f_{j}}$ where $I_{f_{i}} f_{j}$ indicates an interaction term. In principle, we try to keep the number of interactions as small as possible by the choice of the fragments; we have defined, e.g., a fluor atom attached to an aliphatic group to be another fragment than a fluor atom attached to an aromatic group. In this way, we try to limit the number of factors that play a role in the RI prediction. Interactions will be handled in much the same way as fragments. In the remainder of the article, if fragments are mentioned, interactions also apply.

In this article, we describe an approach to find the fragmental values that are used in the calculation of retention indices, based on an analysis of a large set of compounds. Retention indices can be measured easily, in contrast to, e.g., $\log P$ values. Also, they are more reliable than capacity factors. Therefore, they constitute a good and practical measure of polarity. The estimated retention index of a compound can be used to obtain a first guess of a suitable mobile phase. Results show that the predicted mobile phase

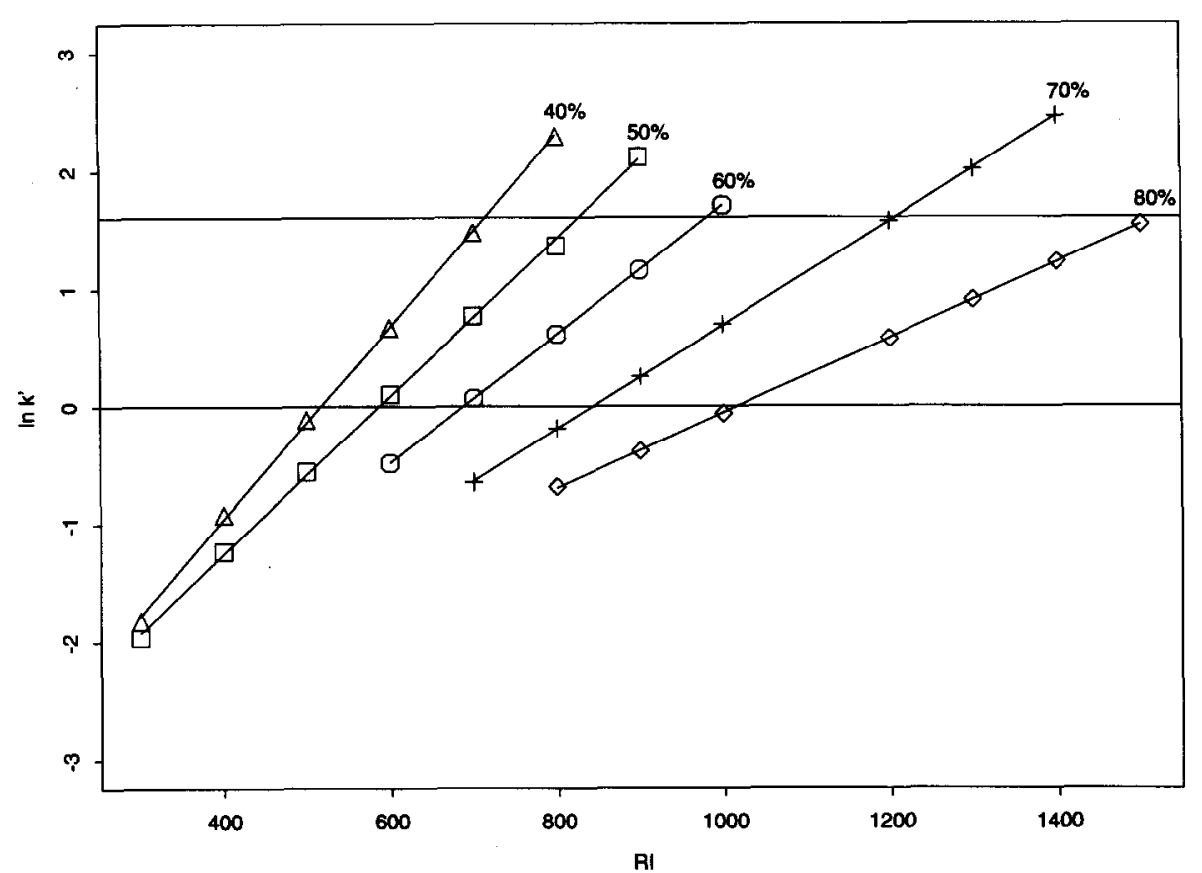

Fig. 2. Relation between the natural logarithm of the capacity factor, the retention index and modifier percentage for 2-ketoalkanes. 
composition is robust against errors in the prediction of retention indices. Another advantage of the method is the easy calibration procedure.

In the next section, some related work will be discussed. After that, our data sets and the actual determination of the fragmental values will be discussed. A method based on the functional validation of the system is used to obtain optimal fragmental values. Results of retention index prediction as well as mobile phase composition prediction will be given.

\section{Related work}

Numerous approaches have been published to infer chemical properties such as hydrophobicity from chemical structure. A global division can be made in methods that use the molecular structure as a whole, such as methods using topological indices [1], and methods that separate the molecule into fragments that account for distinct chemical functionalities (see, e.g., Refs. [8-12]). In the prediction of HPLC parameters, the latter group of methods is the most popular. Also combinations of the two are reported (see below). Although some approaches for the prediction of retention as well as suitable mobile phase compositions in RP-HPLC have been published, to the knowledge of the authors, the explicit combination of retention index prediction and optimal mobile phase composition prediction is new.

\subsection{Mobile phase composition prediction}

Several systems have been described in the literature that predict a mobile phase composition for a good separation [13-15]. Most approaches divide the structure of interest into fragments. Hindriks et al. [13] attach a percentage of methanol to each fragment, and the sum of the percentages then gives a first estimate of the percentage for the complete molecule. Compounds have been collected from a data base of central nervous system (CNS) active drugs. The predictions have been performed for two types of columns and for two $\mathrm{pH}$ values, with a mobile phase containing methanol as organic modifier.
The system also advises on new conditions if the first guess was unsatisfactory (Second and Third Guess). After the third guess, very good results have bcen obtained. However, the number of different fragments that can be used in the prediction is rather small. This, of course, limits the applicability of the system [16].

In another approach [14], the mobile phase composition for a mixture of compounds is calculated from the octanol-water partition coefficients $(\log P)$ of the compounds. Again, fragmental constants are used to calculate the parameter of interest, $\log P$. The advantage of using $\log P$ as a predictor for mobile phase composition is that for many fragments, $\log P$ values are known. Amongst others, Rekker [9] and co-workers have developed a method to predict $\log P$. However, this method can be rather complex and a wrong choice of individual fragment values and correction terms may lead to large prediction errors. Another disadvantage is that it is difficult and time-consuming to determine $\log P$ values experimentally. Furthermore, although correlations between retention and $\log P$ are often quite good if one class of chemicals is used, predictions are often not very reliable [1] in case of a wider group of compounds. However, good results are reported, also in case of mixtures [14]. Similar rules for the Second and Third Guess are used as in the above approach. Both acetonitrile and methanol can be used as organic modifiers.

Hamoir et al. [15] investigated the usefulness of predicted or measured $\log P$ values for predicting mobile phase compositions. Predictions of $\log P$ values are performed by the same method as above [9]. It was found that predictions were, although not always perfect, satisfactory for a first guess system. If no $\log P$ values can be predicted, the total number of carbon atoms in the molecule may also be used to obtain a first guess.

\subsection{Prediction of retention parameters}

A number of groups have been involved in the prediction of retention parameters from structural data (see, e.g., Refs. [17-23]). Here, some approaches use molecular parameters such as 
topological descriptors as well as fragmental methods to predict capacity factors [19]. Although for most factors significant correlations can be found, the dominant one in most cases is the hydrophobicity.

Some groups predict retention indices rather than retention times or capacity factors. Hasan and Jurs [20] predict retention indices using 17 descriptors, divided into topological descriptors such as the number of aromatic rings, geometrical descriptors such as the largest principal axis, and physical property descriptors, such as the $\log P$ partition coefficient. Eventually, linear equations with four and five variables are found. Polyaromatic hydrocarbons were used as reference compounds. Moreshita et al. [23] predict retention indices of substituted benzenes with $n$-alkanes as reference compounds. Four fragments and a small number of interaction terms, depending on the position of the substitution, are defined. Very good agreement with experimental values is found, but the range of molecules for which a prediction can be made is quite small. Smith and Burr [17] define fragmental retention index increments whose values are related quadratically to the amount of organic modifier. The retention index of molecules containing a phenyl group, the 'parent compound', and any of these fragments now can be predicted for any modifier percentage. This takes into account that retention indices may be dependent on the polarity of the mobile phase. Each fragmental value is determined by three coefficients that are determined by analysis of homologous series derived from the parent compound. Values for coefficients of both fragmental increments and interaction terms have been included in an expert system $[17,18]$. Retention indices are based on alkyl aryl ketones as reference compounds, and both methanol and acetonitrile have been used as modifiers.

Recently, also neural networks [24] have been used to predict retention indices in liquid chro- matography [25]. Similar data as in the present article have been used to test molecular representations that are suitable for the neural network. One of the representations was the fragmental representation also employed here, other representations are based on connectivity indices. For the results, the reader is referred to Ref. [25].

\section{Retention index prediction from fragmental values}

The main problem in the prediction of retention indices from structural fragments is to assign a correct value to each fragment. Multiple regression will in many cases lead to chemically irrelevant results, and in our experience performs badly when subjected to cross-validation procedures. Possible reasons for this include the relatively small size of the data sets employed compared with the number of dependent variables used in the prediction, and the use of homologous series in the calculation of fragment values. Therefore, we decided to use an already existing data base of compounds that did not form a homologous series (see below) to derive fragment values. In this section, RIPS (retention index prediction system), the expert system developed to optimise fragmental constants for RI prediction, is discussed.

First, the molecule, represented by a connection table, is divided into fragments. This is done by a computer program running on a personal computer. Fragments are defined in a small data base containing simple rules. The rule for the fragment $\mathrm{OH}$-arom is (somewhat translated): "find an oxygen that is attached to one aromatic group and one hydrogen atom". At present, our fragment set contains approximately one hundred fragments. After the division of a molecule into fragments, these are read into the expert system. Two major modes exist (see Table 1). If all fragmental constants are known, the RI can be pre-

Table 1

Expert system modes. RIM stands for molecular retention index, RIF for fragment or interaction retention index

\begin{tabular}{llll}
\hline Mode & RIM & RIF & Action \\
\hline $\begin{array}{l}\text { Prediction mode } \\
\text { Refinement mode }\end{array}$ & Unknown & Known & Trivial addition of fragment and interaction values \\
Known & Unknown & Intelligent search to find fragment and interaction values \\
\hline
\end{tabular}


dicted and from that the optimal percentage of methanol can be found using the graph of Fig. 1 . As this mode is trivial, we will in the remainder of the article concentrate on the second mode. This mode is activated when no precise values for the fragmental retention indices are known. In this case, the system tries to derive good values for fragments, based on trial values and intelligent search strategies. The retention indices of the molecules must be known in advance, since they represent the criterium by which the set of fragmental values is judged. Boundaries and initial values were obtained from literature, either from published retention index values [26-29], or from correlations with other physicochemical descriptors $[9,8]$.

Analysis of the results with the trial values for the fragments leads the expert system to propose changes in the fragmental constants. These changes may be incorporated automatically by the system, so that an autonomous search for the best solution may be performed, or they may be selected by the user, thus giving the user the opportunity to incorporate his chemical intuition. A second advantage of the latter possibility is that the user can see what reasons the system has for adapting fragments. The approach implemented in RIPS has been applied in several expert systems in medicine [30,31] and chemistry [32], and enables users to optimise or tailor an expert system to their specific needs.

In general, as many molecules as possible are treated at the same time. The refinement approach uses statistical measures to identify fragments and interactions whose values are likely to be incorrect. The eventual fragment value will be more reliable if the fragment is present more often in the data set.

\subsection{Refinement of fragmental RI values (RIF val- ues)}

The strategy used in the refinement is actually very simple. After the system has calculated RI values for all molecules using a trial set of fragmental values (fragmental RI values will hereafter be indicated by RIF), the difference is calculated between the true RI and the predicted value for each molecule. Then the system decides whether the difference is acceptable or not. In case the predicted value was too high, the error is called false positive (FP); if the predicted value was too low, the error is called false negative (FN). The amount with which a predicted RI may differ from the true RI can be set by the user. The number of times a fragment or interaction is used in an incorrect prediction, and the size of the errors, is taken into account when calculating refinements for the fragment. However, each proposed refinement should fall within the specified boundaries for the fragment. Three types of refinements are implemented: one-way refinements, RMS-improving refinements, and corrective refinements. They will be described briefly below.

\subsubsection{One-way refinements}

One-way refinements are applicable if a fragment is present only in FN or FP molecules (not both, and not in correctly predicted molecules). The RIF value of such a fragment will be adapted so that most molecules become correct, and none are overcorrected. Therefore, no FP molecule may become FN and vice versa; they only may become less FP or correct. This is shown in Fig. 3. After one or more rounds of refinements, generally all fragments will be used in some correct predictions, and this refinement type can no longer be used.

\subsubsection{RMS-improving refinements}

RMS-improving refinements can be selected when no one-way refinements are found. They aim at minimizing the root-mean-square (RMS) of the prediction by univariately changing fragment values. The RMS is given by

$\mathrm{RMS}=\sqrt{\frac{\sum_{i}^{N}\left(\mathrm{RIM}_{i}-\mathrm{RI}_{i}\right)^{2}}{N}}$

where $N$ is the number of molecules, $\operatorname{RIM}_{i}$ and $\mathrm{RI}_{i}$ are the predicted and true retention indices of molecule $i$, respectively. The results of an RMS-improving refinement are also depicted in Fig. 3. The difference with one-way refinements is clear from this figure: RMS-improving refine- 
0

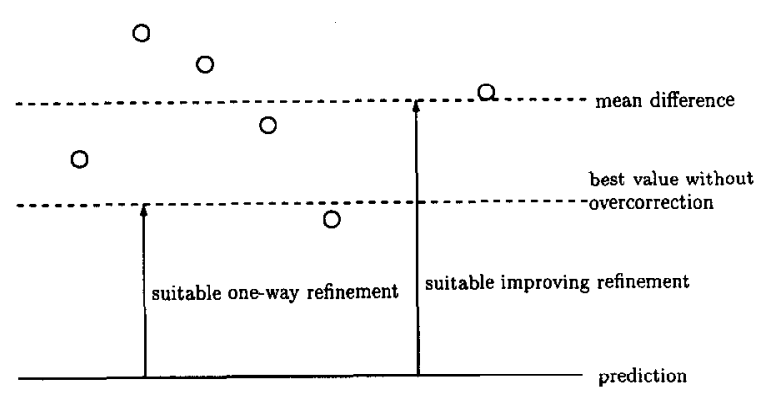

Fig. 3. One-way refinement and RMS-improving refinement of a FN prediction. The solid line indicates the predicted values of a number of molecules containing the same fragment, whereas the true values are indicated by the circles. One-way refinement will increase the value of the fragment with a smaller amount than the RMS-improving refinement to prevent an overcorrection.

ments ignore the fact whether predictions are correct or not, and therefore can make FN molecules FP and vice versa; however, the RMS of the prediction will always decrease when using this type of refinement. Whether or not an individual prediction is FN or FP is of lesser importance than the performance on the whole data set.

\subsubsection{Corrective refinements}

Corrective predictions are possible if a fragment value can be changed in such a way that no correct predictions become incorrect, and one or more incorrect predictions become correct. This type of refinement has only a limited effect on the overall prediction of the data set and is in most cases used to prevent the univariate search of the RMS-improving refinements to get stuck in a local optimum.

\subsubsection{Refinements in practice}

The user who wants to refine the RIF values for the fragments in his data set can do so very easily. A training set has to be defined in which the true RI values for all molecules are known. Then, the predicted RI values are compared with the true values and the expert system proposes several refinements. In general, one-way refine- ments should be selected immediately, since they represent corrections to important errors in the RIF values. Depending on the quality of the prediction, the user may decide to let the system refine itself automatically. This is especially useful when a set of fragments is trained for the first time and boundaries are still spacious. In case the fragments already have been refined before, manual optimisation is a better option. This continues until no further improvement can be observed, or until the user is satisfied with the results.

\section{Data}

A large data set consisting of structural fragments of 350 molecules with known retention index has been obtained from Organon. All molecules are CNS-active drugs, but do not belong to a homologous series. The molecules in the data set contain 84 different fragments and three interactions. From this large set, smaller sets have been created to ensure that all fragments are at least three times present in a data set. Molecules that contain fragments that do not comply with this criterium have been excluded. Two data sets have been analysed: one small data set (set A) consisting of 56 molecules containing 23 fragments and one interaction, measured at $60 \%$ methanol $(\mathrm{MeOH})$, and a larger set (set $\mathrm{B}$ ) measured at methanol percentages between $55 \%$ and $65 \%$ (inclusive). Set B, containing 38 fragments and 2 interactions, includes set $A$ and contains 113 molecules. By selecting data sets on the basis of the modifier percentage at which retention indices have been measured, the effects of the modifier percentage on the retention index are excluded. As a result, the predicted RI for a molecule in the data set must be interpreted as the RI at $60 \% \mathrm{MeOH}$.

Fragments and interactions that are present in set $\mathrm{A}$ have been gathered in Table 2. Also boundaries and the number of times the fragments are present in the data sets have been included. Fragments of set B that are not present in set A are gathered in Table 3. Initial values were obtained from literature, either from published retention 
Table 2

Fragments present in both sets, $A$ and $B$

\begin{tabular}{|c|c|c|c|c|}
\hline Fragment & $\begin{array}{l}\text { No. of } \\
\text { occur- } \\
\text { rences } \\
\text { set A }\end{array}$ & $\begin{array}{l}\text { No. of } \\
\text { occur- } \\
\text { rences } \\
\text { set B }\end{array}$ & Up & Low \\
\hline$\overline{\mathrm{CH}_{3}}$ & 82 & 139 & 99 & 75 \\
\hline $\mathrm{CH}_{2}$ & 204 & 443 & 100 & 100 \\
\hline $\mathrm{CH}^{2}$ & 57 & 118 & 106 & 66 \\
\hline $\mathrm{C}$ & 5 & 17 & -10 & -31 \\
\hline $\mathrm{C}_{6} \mathrm{H}_{5}$ & 19 & 48 & 506 & 471 \\
\hline $\mathrm{C}_{6} \mathrm{H}_{4}$ & 66 & 111 & 545 & 509 \\
\hline $\mathrm{C}_{6} \mathrm{H}_{3}$ & 10 & 29 & 643 & 577 \\
\hline $\mathrm{C}_{6} \mathrm{H}_{2}$ & 6 & 6 & 620 & 536 \\
\hline $\mathrm{C}_{5} \mathrm{II}_{x} \mathrm{~N}$ & 4 & 8 & 443 & 373 \\
\hline aliph-NH-aliph & 5 & 14 & -395 & -449 \\
\hline aliph-N-2aliph & 46 & 84 & -363 & -391 \\
\hline aliph-N-aliph-arom & 15 & 24 & -132 & -172 \\
\hline aliph-N-2arom & 4 & 5 & -62 & -162 \\
\hline $\mathrm{HON}=\mathrm{C}-\mathrm{NH}_{2}$ & 4 & 4 & -462 & -525 \\
\hline arom-O-arom & 13 & 18 & -90 & -150 \\
\hline aliph-O-arom & 13 & 20 & -52 & -124 \\
\hline aliph-O-aliph & 6 & 7 & -54 & -100 \\
\hline $\mathrm{OH}$-aliph & 6 & 19 & -42 & -62 \\
\hline $\mathrm{Cl}$-arom & 6 & 21 & -259 & -319 \\
\hline $\mathrm{N}=\mathrm{C}-\mathrm{N}$ & 4 & 6 & -180 & -300 \\
\hline$x$ arom $-\mathrm{C}=\mathrm{C}-\mathrm{xarom}$ & 5 & 6 & 27 & -9 \\
\hline aliph $-\mathrm{C}=\mathrm{C}-\mathrm{xarom}$ & 3 & 7 & 132 & 112 \\
\hline vicinal-O-interaction & 7 & 11 & 135 & -21 \\
\hline
\end{tabular}

$\mathrm{C}_{6} \mathrm{H}_{5}$ designates a monosubstituted phenyl. An ' $x$ ' in the name of a fragment indicates a variable number. The fragment aliph $-\mathrm{C}=\mathrm{C}-x$ arom for instance, contains two carbon atoms connected with a double bond; one of the carbon atoms is connected to at least one aromatic ring, the other only to aliphatic components. Hydrogens may or may not be explicitly mentioned in the fragment names; if not, they are considered to be aliphatic. 'Up' and 'Low' indicate the upper and lower boundaries for the RIF values, respectively.

index values [26-29], or from correlations with other physicochemical descriptors [8,9].

\section{Results of the refinement approach}

\subsection{Retention index prediction}

Retention index predictions for the two data sets have been optimised using the refinement mode of RIPS. The RMS values for the training on the complete sets were 104 and 162 for sets A and $B$, respectively. Small differences in RMS values may be found in different optimization runs. In every optimization run, one-way refinements have been applied whenever possible. For the rest, automatic refinement mode has been used to reach an optimum. Only in the last stage of the optimization corrective refinements have been used to prevent the system from being trapped in a local optimum. This, however, did not cause large deviations in fragmental values.

Analysis of the results showed that many false positive predictions were made for molecules that contained three or more aromatic rings. Therefore, optimization runs have been done on both data sets in which an extra interaction was taken into account: multiple-arom-interaction. These extended data sets will be denoted $\mathrm{A}+$, containing two times, and $\mathrm{B}+$, containing eight times this interaction. Significant improvements in RMS values were obtained. The best RMS for set $A+$ was 87 , and for set $B+141$. The physicochemical rationale behind this interaction is that the third aromatic ring in a molecule will not have the same effect on the retention as the first aromatic ring. It is possible to define this kind of interaction for more fragments, but the danger is

Table 3

Fragments present in set B, but not in set $A$. The fusedarom-interaction is present in a molecule if two aromatic rings share two atoms

\begin{tabular}{llrr}
\hline Fragment & $\begin{array}{l}\text { No. of } \\
\text { occur- } \\
\text { rences } \\
\text { set B }\end{array}$ & Up & Low \\
& 10 & 129 & \\
\hline F-arom & 3 & 280 & -120 \\
$\mathrm{CF}_{3}$-arom & 6 & -50 & -200 \\
OH-arom & 5 & -300 & -600 \\
arom=O & 3 & 187 & -13 \\
arom-S-arom & 5 & 100 & -100 \\
arom-aliph-C=O & 3 & -200 & -600 \\
arom-CON-2aliph & 5 & -63 & -464 \\
aliph-CON-aliph-arom & 5 & -225 & -625 \\
aliph-CONH-aliph & 7 & -104 & -361 \\
$\mathrm{NH}_{2}-$ aliph & 6 & -277 & -330 \\
aliph-COO-aliph & 3 & -66 & -266 \\
$x$ arom-C=N-aliph & 4 & 466 & 266 \\
$\mathrm{C}_{3} \mathrm{H}_{x} \mathrm{~N}_{2}$ & 5 & 537 & 137 \\
$\mathrm{C}_{4} \mathrm{H}_{x} \mathrm{~N}$ & 3 & 766 & 366 \\
$\mathrm{C}_{4} \mathrm{H}_{x} \mathrm{~N}_{2}$ & 8 & 0 & -200 \\
fused-arom-interaction & & & \\
\hline
\end{tabular}


that too many descriptors are being used in the retention index prediction. Therefore, we chose to add only the most obvious one.

Furthermore, a cross-validation procedure has been performed on the data of sets $\mathrm{A}$ and $\mathrm{A}+$. In this procedure, a test set containing three molecules is excluded from the data set, and optimal fragment values are obtained by training the system on the remainder (i.e., the training set). Then, the results of the RI prediction on the three excluded molecules are used to assess the quality of the prediction. By excluding all molecules in random order, a cross-validation RMS value can be calculated. For sets $A$ and $\mathrm{A}+$, these values are 123 and 111, respectively. Cross-validation RMS values are (of course) somewhat higher than training RMS values, but the differences are small enough to have confidence in the fragment values that are derived. Fragment values did not show a large variance in the different optimizations, nor did mean and median values differ very much. Mean fragmental values are used as optimal values for the fragments in sets $\mathbf{A}$ and $\mathbf{A}+$, and are given in Table 4. As can be seen, the addition of the multiplearom-interaction did not influence the values found for other fragments very much. Predictions for the two sets with the mean values of the cross-validation procedures are depicted in Fig. 4.

Using the optimised values for the fragments present in set $\mathbf{A}+$, the next step was to optimise the values for the fragments that were present only in set B. Values of fragments present in set A could also be refined, but in most cases this was not necessary. No cross-validation procedure was performed for the fragments in set $\mathrm{B}$, since most of them were only present less than six times. The values found in the training of the complete sets B and B + are gathered in Table 5 . Predictions with these values are depicted in Fig. 5.

When comparing the predictions of sets $\mathrm{A}$ and $A+$ on one hand and sets $B$ and $B+$ on the other, the results of the sets with the multiplearom-interaction are clearly better. In the sets without this interaction, other fragments try to compensate for the false positive predictions that arise from two or more aromatic rings, causing
Table 4

Optimal retention indices of fragments in sets $\mathrm{A}$ and $\mathrm{A}+$, derived from cross-validation

\begin{tabular}{|c|c|c|}
\hline Fragment & RIF set $A$ & RIF set $\mathrm{A}+$ \\
\hline $\mathrm{CH}_{3}$ & 87 & 86 \\
\hline $\mathrm{CH}_{2}$ & 100 & 100 \\
\hline $\mathrm{CH}^{2}$ & 84 & 82 \\
\hline $\mathrm{C}$ & -32 & -31 \\
\hline $\mathrm{C}_{6} \mathrm{H}_{5}$ & 486 & 489 \\
\hline $\mathrm{C}_{6} \mathrm{H}_{4}$ & 528 & 527 \\
\hline $\mathrm{C}_{6} \mathrm{H}_{3}$ & 616 & 610 \\
\hline $\mathrm{C}_{6} \mathrm{H}_{2}$ & 575 & 563 \\
\hline $\mathrm{C}_{5} \mathrm{H}_{x} \mathrm{~N}$ & 404 & 401 \\
\hline aliph-NH-aliph & -442 & -420 \\
\hline aliph-N-2aliph & -377 & -378 \\
\hline aliph-N-aliph-arom & -144 & -138 \\
\hline aliph-N-2arom & -115 & -112 \\
\hline $\mathrm{HO}-\mathrm{N}=\mathrm{C}-\mathrm{NH}_{2}$ & -463 & -462 \\
\hline arom-O-arom & -119 & -99 \\
\hline aliph-O-arom & -88 & -88 \\
\hline aliph-O-aliph & -111 & -62 \\
\hline $\mathrm{OH}$-aliph & -288 & -293 \\
\hline $\mathrm{Cl}$-arom & 92 & 87 \\
\hline $\mathrm{N}=\mathrm{C}-\mathrm{N}$ & -243 & -241 \\
\hline$x$ arom $-\mathrm{C}=\mathrm{C}-x$ arom & 8 & 9 \\
\hline aliph $-\mathrm{C}=\mathrm{C}-\mathrm{x}$ arom & 122 & 122 \\
\hline vicinal-O-interaction & 52 & 51 \\
\hline multiple-arom-interaction & - & -290 \\
\hline
\end{tabular}

Values in the second column are derived without multiplearom-interaction (set $\mathrm{A}$ ), values in the third column are derived with multiple-arom-interaction (set $\mathrm{A}+$ ). The vicinal-O-interaction is present in molecules in which two oxygen atoms are attached to vicinal atoms. Only one of the two oxygens may be part of a ring.

extra false negative situations. Including the multiple-arom-interaction thus not only reduces the number of FP errors, but also the number of FN errors.

This illustrates a fundamental problem in the approach using fragments: which interactions must be taken into account, and which interactions can be subsumed by defining larger fragments? One should define fragments in such a way that a reasonably good prediction for $\mathbf{R I}$ values can be obtained without 'overtraining'. Furthermore, one should be aware that several fragments can exhibit aberrant behaviour under different circumstances. The contribution of nitrogen-containing fragments to retention behaviour, for instance, in many cases is strongly pH-dependent. 
$\mathrm{RI}$ prediction set $\mathrm{A}$

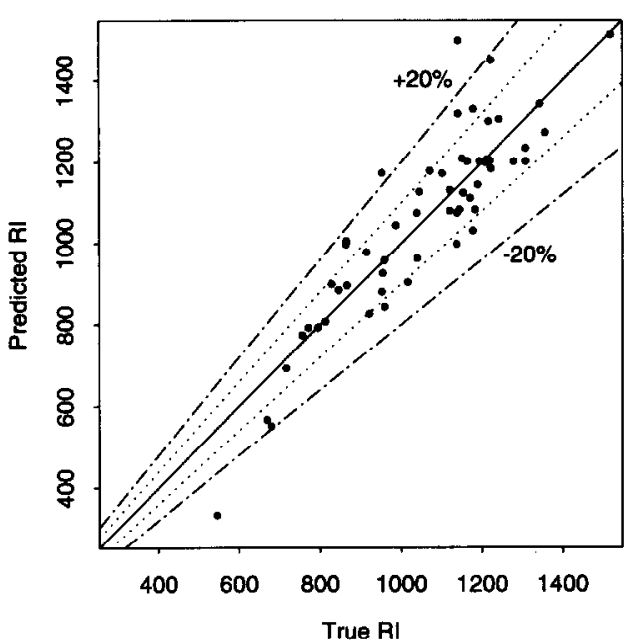

RI prediction set $\mathbf{A +}$

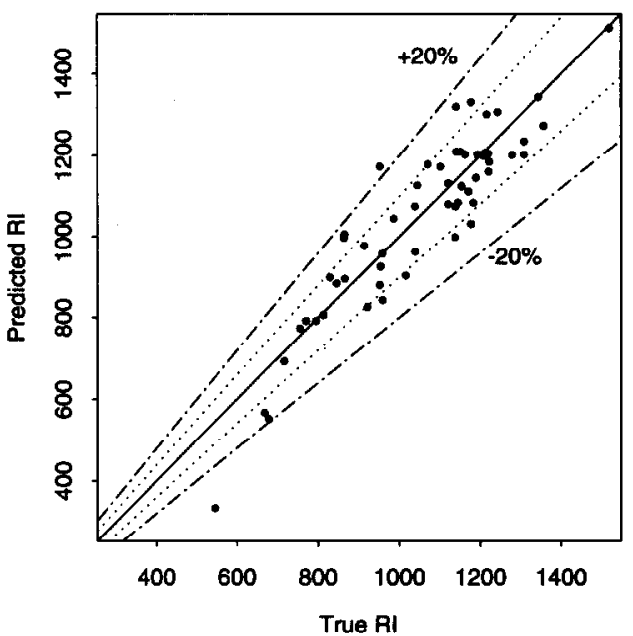

Fig. 4. True versus predicted retention indices for sets $\mathrm{A}$ and $\mathrm{A}+$.

\subsection{Prediction of mobile phase composition}

Using the fragmental values for fragments in set $\mathrm{A}+$, for a small group of 17 molecules, the mobile phase composition was predicted for which a capacity factor of 5 would be found. The 17 test compounds are given in Table 6; experimental results are gathered in Table 7 . The predicted percentage of $\mathrm{MeOH}$ in all cases was rounded to a multiple of $5 \%$. In 13 out of 17 cases, the capacity factor found by the percentage of $\mathrm{MeOH}$ predicted by the expert system was in the range between 3 and 10; in 9 out of 17 cases the capacity factor was in the range between 4 and 7 . The mean capacity factor found for the 17 compounds was 5.2 , and the standard deviation was 2.5 , largely because one compound (compound 1) was found to have a capacity factor of 12.7 at the predicted modifier percentage. The cause for this was a retention index that was predicted too low with almost 200 units. Such errors in the prediction of retention indices may be expected, because of the simplicity of the model that is used. Nevertheless, the results of a false classification do not always have the same effect (see compound 14). The apparent bias in the predictions
(RI values are mostly predicted too low) was (of course) absent in the training sets.

These results indicate that the RI prediction is

Table 5

Optimal retention indices of fragments only present in sets $B$ and $B+$, derived by training. Optimal values of fragments in both sets $\mathrm{B}$ and $\mathrm{B}+$ were approximately equal for all fragments

\begin{tabular}{lc}
\hline Fragment & RIF sets B $/ \mathrm{B}+$ \\
\hline F-arom & -51 \\
$\mathrm{CF}_{3}$-arom & 80 \\
OH-arom & -100 \\
arom=O & -412 \\
arom-S-arom & -6 \\
arom-aliph-C=O & 0 \\
arom-CON-2aliph & -339 \\
aliph CON-aliph-arom & -439 \\
aliph-CONH-aliph & -358 \\
$\mathrm{NH}_{2}-$ aliph & -114 \\
aliph-COO-aliph & -277 \\
$x$ arom-C=N-aliph & -66 \\
$\mathrm{C}_{3} \mathrm{H}_{x} \mathrm{~N}_{2}$ & 466 \\
$\mathrm{C}_{4} \mathrm{H}_{x} \mathrm{~N}$ & 257 \\
$\mathrm{C}_{4} \mathrm{H}_{x} \mathrm{~N}_{2}$ & 766 \\
fused-arom-interaction & -100 \\
\hline
\end{tabular}


RI prediction set $B$

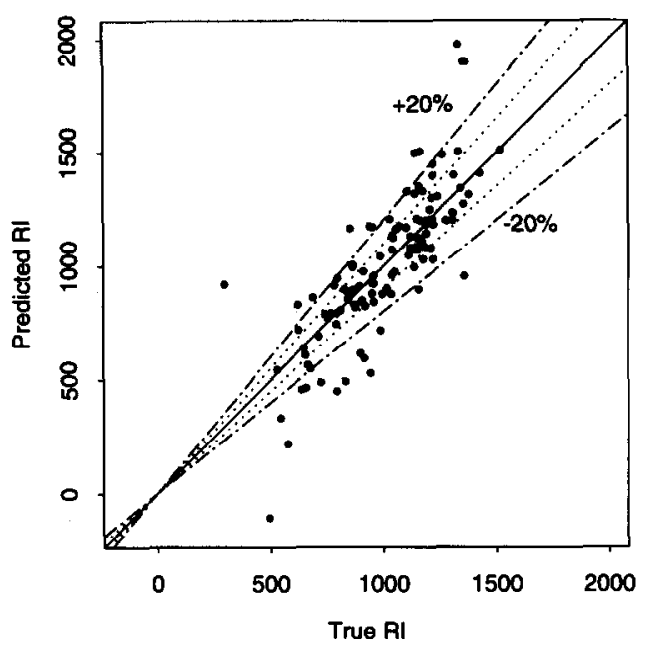

RI prediction set $B+$

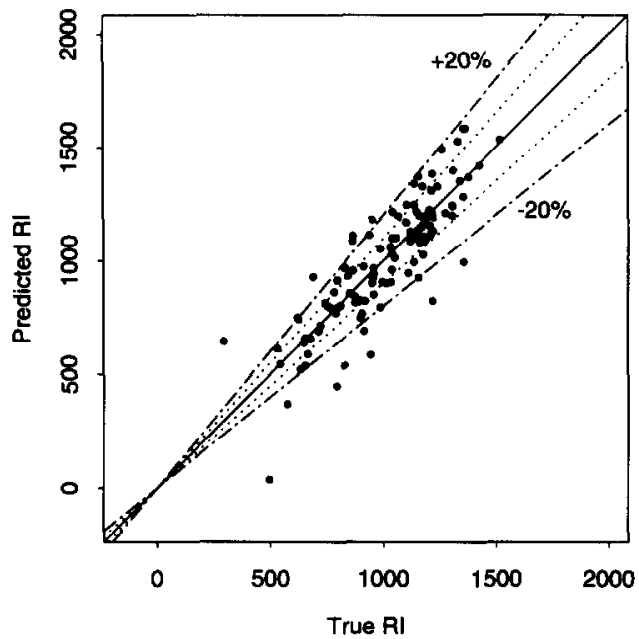

Fig. 5. True versus predicted retention indices for sets $B$ and $B+$.

satisfactory if used for the prediction of mobile phase compositions. As can be seen in Fig. 1, the capacity factor of compounds with a large reten- tion index is less sensitive to changes in mobile phase composition. Rather large errors may be present in the prediction of the retention index

Table 6

The composition of the 17 test compounds. The numbers indicate the number of occurrence of the fragments. In total, 183 fragments are present in the 17 molecules

\begin{tabular}{|c|c|c|c|c|c|c|c|c|c|c|c|c|c|c|c|c|c|}
\hline \multirow[t]{2}{*}{ Fragment } & \multicolumn{17}{|c|}{ Compound } \\
\hline & 1 & 2 & 3 & 4 & 5 & 6 & 7 & 8 & 9 & 10 & 11 & 12 & 13 & 14 & 15 & 16 & 17 \\
\hline $\mathrm{CH}_{3}$ & 2 & 1 & 1 & 2 & 4 & & 2 & & 1 & & 1 & 1 & 3 & & 1 & & 1 \\
\hline $\mathrm{CH}_{2}$ & 3 & 3 & 3 & 7 & 2 & 5 & 8 & 2 & 3 & 8 & 2 & 2 & 4 & 4 & 4 & 4 & 2 \\
\hline $\mathrm{CH}$ & & 2 & 1 & 1 & & 1 & 2 & 2 & & & 1 & 1 & 2 & & & & 1 \\
\hline $\mathrm{C}$ & & & & & 1 & & & & & 1 & & & & & & & \\
\hline $\mathrm{C}_{6} \mathrm{H}_{5}$ & 2 & & & 1 & & & 2 & & & 2 & & & & & & & 1 \\
\hline $\mathrm{C}_{6} \mathrm{H}_{4}$ & & 2 & 2 & 1 & 2 & 2 & & 2 & 2 & & 1 & 1 & 1 & 1 & 2 & 1 & \\
\hline $\mathrm{C}_{6} \mathrm{H}_{3}$ & & & & & & & & & & & 1 & 1 & & 1 & & 1 & 1 \\
\hline aliph-NH-aliph & & & & & & & & 1 & & & 1 & 1 & & 1 & & & \\
\hline aliph-N-2aliph & 1 & 1 & 1 & 1 & 1 & & 1 & & 1 & 1 & & 1 & & & 1 & 1 & 1 \\
\hline aliph-N-aliph-arom & 1 & 1 & 1 & 1 & & 1 & 1 & & & & & & & & & & \\
\hline arom-O-arom & & & & & & & & 1 & 1 & & 1 & 1 & & 1 & 1 & 1 & 1 \\
\hline aliph-O-arom & & & & & & & & & & & & & 1 & & & & \\
\hline aliph-O-aliph & & & & & & & 1 & & & & & & 1 & & & & \\
\hline $\mathrm{OH}$-aliph & & 1 & & & & & & & & 1 & & & 1 & & & & 1 \\
\hline $\mathrm{Cl}$-arom & & & & & & & & & & & 1 & 1 & & 1 & & 1 & 1 \\
\hline $\mathrm{N}=\mathrm{C}-\mathrm{N}$ & & & & & & & & & & & & & & 1 & & & \\
\hline$x$ arom $-\mathrm{C}=\mathrm{C}-x$ arom & & & & & & & & & 1 & & & & & & & & \\
\hline aliph $-\mathrm{C}=\mathrm{C}-\mathrm{x}$ arom & & & & & 1 & & & & & & & & & & & & \\
\hline vicinal-O-interaction & & & & & & & & & & & & & 1 & & & & \\
\hline
\end{tabular}


Table 7

Results for the 17 test compounds

\begin{tabular}{rrrrr}
\hline Compound & $\begin{array}{l}\text { Predicted RI } \\
(60 \% \mathrm{MeOH})\end{array}$ & $\begin{array}{l}\text { Measured RI } \\
(60 \% \mathrm{MeOH})\end{array}$ & $\% \mathrm{MeOH}$ & $k^{\prime}$ \\
\hline 1 & 930 & 1115 & 55 & 12.7 \\
2 & 797 & 794 & 45 & 6.1 \\
3 & 1003 & 1010 & 60 & 4.7 \\
4 & 1452 & - & 80 & 3.8 \\
5 & 1316 & - & 75 & 4.5 \\
6 & 1493 & - & 80 & 2.4 \\
7 & 1498 & - & 80 & 3.6 \\
8 & 900 & 1063 & 55 & 6.5 \\
9 & 973 & 1019 & 60 & 2.9 \\
10 & 1080 & 1095 & 65 & 5.3 \\
11 & 1078 & 1098 & 65 & 2.7 \\
12 & 1207 & - & 70 & 4.2 \\
13 & 508 & - & 30 & 9.5 \\
14 & 867 & 1072 & 55 & 6.3 \\
15 & 1064 & 1137 & 65 & 3.9 \\
16 & 1107 & - & 65 & 5.3 \\
17 & 879 & 1004 & 55 & 5.1 \\
\hline
\end{tabular}

The second column contains the predicted RI, the third contains the measured $\mathrm{RI}$, if available. From the predicted RI a percentage of modifier is calculated (fourth column). The capacity factor of the compound with the proposed mobile phase composition is given in column five.

while still a valuable mobile phase composition may be expected. Also, out of practical considerations, mobile phase compositions with more than $80 \% \mathrm{MeOH}$, or less than $30 \% \mathrm{MeOH}$ are rarely used. Prediction errors in the extreme retention index range will therefore also have no significant effects on the mobile phase prediction.

\section{Discussion}

In this work, we have used an existing data set to derive retention indices for molecular fragments. These fragmental retention indices are added to obtain an estimate of the retention index of the complete molecule under the conditions pH 7.4, column: $\mu$-Bondapak, mobile phase composition: 60:40 MeOH-water. Apart from the fact that an estimate of the retention index of a compound obtained from the chemical structure alone is valuable in its own right, this prediction can also be used to obtain an estimate of the mobile phase composition at which a good sepa- ration can be expected. The advantage of using retention indices rather than directly predicting capacity factors lies in the greater reproducibility of retention indices.

Retention index prediction using fragmental values has the advantage that it is intuitively appealing. Estimates for newly defined fragments may be made using chemical knowledge. The system presented here makes it possible to interactively refine these fragment values until acceptable results have been obtained. It is very important to define reasonable limits for fragmental values. Cross-validation using multiple linear regression to obtain fragmental values yields very different values in each run. Essentially, too many degrees of freedom will be present to make this procedure reliable. Therefore, the range in which a fragment value may lie must be defined a priori. With these ranges, values derived from crossvalidation procedures are quite reliable. The more compounds are present containing a certain fragment, the more reliable the fragment value will be.

The fragment values are derived using a knowledge-based system, RIPS, that is implemented in KEE 4.0 (Intellicorp, Inc.) running on SUN workstations. New fragment values can easily be obtained when a sufficiently large data set is present. This way, the system can be used to find fragment values for retention on other column types and with other organic modifiers. Effects of ageing columns and column batch-tobatch variations can be taken into account by a simple calibration procedurc, in which a series of reference compounds used in the RI scale is analyzed with different percentages of organic modifier. For everyday practice, a lean version of RIPS lacking the refinement modules and running on personal computers has been written; given a connection table of the compound of interest, the fragment composition, retention index at $60 \% \mathrm{MeOH}$, and the proposed modifier concentration will be written to file.

Fragmental values derived by training must be regarded with caution. Even greater caution must be taken with values that are derived from homologous series, a situation that can often be found in literature. The retention mcchanism in 
reversed-phase HPLC is rather complex and not yet fully understood. Especially the influence of fragments upon each other will cause deviations from the expected behaviour. Therefore, retention index prediction in HPLC is not as reliable as in GC. Nevertheless, the quality of the predictions found in this study is good enough to be able to predict satisfying mobile phase compositions. The advantage of the present system is that the set of fragments can be extended gradually. Values for new fragments, present in a number of compounds with known retention indices can be refined, without having to extensively alter already known values for other fragments.

The method of dividing a molecule into a set of independent fragments and possibly a few interactions also has some disadvantages. First of all, a set of fragments must be defined. This set may not be too large, or else it will be impossible to derive reliablc values for the fragments (cf. drawing a line through two points). On the other hand, it may not be too small because the number of compounds for which the prediction is possible will not be worthwhile. The definition of a set of fragments is always subjective and may not always be correct for the given problem. Furthermore, the values that are to be attached to the fragments are difficult to find, and may not be equal in all situations (interaction effects). This poses an extra constraint on the definition of fragments. Still, despite the simplicity of the model, useful results can be obtained. In this work, using a limited set of fragments RI predictions with RMS values of 90-140 are reported. Although not very precise, this appeared satisfactory if the predicted value was used to obtain an optimal mobile phase composition. Of course, the true value of the fragmental approach can only be found if it is used in practice and compared with other methods. For that, much experimental work will be needed.

\section{Acknowledgements}

The authors would like to thank A. Debets and R. Vervoort of Organon, Oss, for the data and stimulating discussions. W. Oussoren and R. van den Schoor are acknowledged for their work in the definition of fragments and initial values, and $E$. Methorst for the implementation of an early version of the program to transform connection tables into fragments.

\section{References}

[1] R. Kaliszan, Quantitative Structure - Chromatographic Retention Relationships, Wiley, New York, 1987.

[2] J.K. Baker, L.A. Cates, M.D. Corbett, J.W. Huber and D.L. Lattin, Laboratory to laboratory reproducibility of high performance liquid chromatographic retention indices, Joumal of Liquid Chromatography, 5 (1982) 829839.

[3] J.K. Baker and C. Ma, Retention index scale for liquidliquid chromatography, Journal of Chromatography, 169 (1979) 107-115.

[4] M. Bogusz and R. Aderjan, Improved standardization in reversed phase HPLC using 1-nitroalkanes as a retention index scale, Journal of Chromatography, 435 (1988) 43-53.

[5] R.M. Smith, Alkylarylketones as a retention index scale in liquid chromatography, Journal of Chromatography, 236 (1982) 313-320.

[6] R.M. Smith and N. Finn, Comparison of retention index scales based on alkyl-arylketones, alkan-2-ones and 1nitroalkanes for polar drugs on reversed-phase HPLC, Journal of Chromatography, 537 (1991) 51-60.

[7] A. Shalaby, Zs. Budvari-Brani and Gy. Szasz, HPLC retention index scale for nitrogen-bridged compounds, Journal of Liquid Chromatography, 7 (1984) 1133-1150.

[8] C. Hansch and A. Leo, Substituent Constants for Correlation Analysis in Chemistry and Biology, Wiley, New York, 1979.

[9] R.F. Rekker, The Hydrophobic Fragmental Constant: Its Derivatization and Application. A Means of Characterizing Membrane Systems, Elsevier, Amsterdam, 1977.

[10] L.R. Snyder, Classification of the solvent properties of common liquids, Journal of Chromatography, 92 (1974) 223-230.

[11] P. Jandera, Method for characterisation of selectivity in reversed-phase liquid chromatography. I. Derivation of the method and verification of the assumptions, Journal of Chromatography, 352 (1986) 91-110.

[12] R.M. Smith, Functional group contributions to the retention of analytes in reversed-phase high-performance liquid chromatography (Review), Journal of Chromatography $A, 656$ (1993) 381-415.

[13] H. Hindriks, F. Maris, J. Vink, A. Peeters, M. de Smet, D.L. Massart and L. Buydens, Expert system for the selection of initial high-performance liquid chromatographic conditions for the analysis of pharmaceuticals, Journal of Chromatography, 485 (1989) 255-265. 
[14] G. Szepesi and K. Valkó, Prediction of initial HPLC conditions for selectivity optimization in pharmaceutical analysis by an expert system approach, Journal of Chromatography, 550 (1991) 87-100.

[15] T. Hamoir, B. Bourguignon, D.L. Massart and H. Hindriks, Model building for the prediction of initial chromatographic conditions in pharmaceutical analysis using reversed-phase liquid chromatography, Journal of Chromatography, 63 (1993) 43-56.

[16] F. Maris, H. Hindriks, J. Vink, A. Peeters, N. Vanden Driessche and D.L. Massart, Validation of an expert system for the selection of initial high-performance liquid chromatographic conditions for the analysis of basic drugs, Journal of Chromatography, 506 (1990) 211-221.

[17] R.M. Smith and C.M. Burr, Retention prediction of analytes in reversed-phase high-performance liquid chromatography based on molecular structure. V. Cripes (Chromatographic Retention Index Prediction Expert System), Journal of Chromatography, 485 (1989) 325-340.

[18] C.M. Burr and R.M. Smith, Retention prediction in RP-HPLC using a functional group database and expert system (CRIPES), Analytical Processes, 26 (1989) 24-26.

[19] K. Jinno and K. Kawasaki, Computer-assisted retention prediction system for reversed-phase micro high-performance liquid chromatography, Journal of Chromatography, 316 (1984) 1.

[20] M.N. Hasan and P.C. Jurs, Computer-assisted prediction of liquid chromatography retention indexes of polycyclic aromatic hydrocarbons, Analytical Chemistry, 55 (1983) 263-269.

[21] P. Jandera, Method for characterisation of selectivity in reversed-phase liquid chromatography. II. Possibilities for the prediction of retention data, Journal of Chromatography, 352 (1986) 111-126.

[22] K. Valkó, G. Szabó, J. Röhricht, K. Jemnitz and F. Darvas, Prediction of retention of metabolites in HPLC by an expert system approach, Journal of Chromatography, 485 (1989) 349-363.

[23] F. Morishita, H. Kakihana and T. Kojima, Measurement of retention indexes on $n$-alkane scale in reversed-phase liquid chromatography and their prediction from molecular structures, Analytical Letters, 17 (1984) 2385-2398.

[24] D.E. Rumelhart and J.L. McClelland, Parallel Distributed Processing. Explorations in the Microstructure of Cognition, Vol. 1. Foundations, MIT, London, 1986.

[25] J.R.M. Smits, W. Melssen, G.J. Daalmans and G. Kateman, Using molecular representations in combination with neural networks. A case study: prediction of the HPLC retention index, Computers and Chemistry, (1993) in press.

[26] R.M. Smith and C.M. Burr, Retention prediction of analytes in reversed-phase high-performance liquid chromatography based on molecular structure. I. Monosubstituted aromatic compounds, Journal of Chromatography, 475 (1989) 57-74.

[27] R.M. Smith and C.M. Burr, Retention prediction of analytes in reversed-phase high-performance liquid chromatography based on molecular structure. III. Monosubstituted aliphatic compounds, Journal of Chromatography, 481 (1989) 71-84.

[28] R.M. Smith and C.M. Burr, Retention prediction of analytes in reversed-phase high-performance liquid chromatography based on molecular structure. IV. Branched and unsaturated alkyl benzenes, Journal of Chromatography, 481 (1989) 84-95.

[29] R.M. Smith and C.M. Burr, Retention prediction of analytes in reversed-phase high-performance liquid chromatography based on molecular structure. VI. Disubstituted aromatic compounds, Journal of Chromatography, 550 (1991) 335-356.

[30] P.G. Politakis and S.M. Weiss, Using empirical analysis to refine expert systems knowledge bases, Artificial Intelligence, 22 (1984) 23-48.

[31] A. Ginsberg, Automatic Refinement of Expert System Knowledge Bases, Pitman, London, 1988.

[32] R. Wehrens, L. Buydens and G. Kateman, Validation and refinement of expert systems: interpretation of NMR spectra as an application in analytical chemistry, Chemometrics and Intelligent Laboratory Systems, 12 (1991) 5768. 